EPJ Web of Conferences 45, 01089 (2013)

DOI: $10.1051 /$ epjconf/20134501089

(c) Owned by the authors, published by EDP Sciences, 2013

\title{
Wall Shear Stress Induced by Taylor Bubbles in Inclined Flow Channels
}

\author{
J. Tihon ${ }^{\text {a }}$, V. Pěnkavová, and J. Vejražka \\ Institute of Chemical Process Fundamentals, Rozvojová 135, 16502 Prague, Czech Republic
}

\begin{abstract}
The motion of single air bubbles in flat channels is experimentally investigated. The electrodiffusion technique of near-wall flow diagnostics is applied to measure the wall shear stress distribution under large rising bubbles. The measurements are synchronized with the visual observation of bubble movement by a high-speed camera. The analysis of video records provides information on the bubble shape and terminal velocity. The experiments are carried out for three different channel configuration (with heights of $1.5,4$, and $8 \mathrm{~mm}$ ), cover a wide range of channel inclination angles (from horizontal to vertical position), and dealing with both the bubbles in stagnant and in co-flowing water. The directionally sensitive, two-strip electrodiffusion probe is proved to be an effective tool to investigate the near-wall flow response to translating bubbles. It provides information not only on the wall shear rate distribution, but also detects the location of near-wall flow reversal, gives an estimate of the thickness of liquid film separating the large bubble from the wall, and provides also the characteristics of capillary waves appearing in the bubble tail region. The effect of channel inclination angle on the modification of wall shear stress distribution along the upper and bottom wall is also discussed.
\end{abstract}

\section{Introduction}

The motion of large bubbles in vertical tubes received considerable attention since the pioneer paper of Dumitrescu [1]. The comprehensive experimental studies on long bubbles in cylindrical tubes done by White and Beardmore [2], Nicklin et al. [3] and Zukolski [4] identified the different regimes of bubble propagation depending on three dimensionless groups: Froude $(\mathrm{Fr}=$ $\left.U_{B} /(g D)^{1 / 2}\right)$, Eötvös $\left(E o=\rho g D^{2} / \sigma\right)$, and Morton $(M o=$ $\left.\left(g \mu^{4}\right) /\left(\rho \sigma^{3}\right)\right)$ number, where $\sigma$ is surface tension, $\mu$ and $\rho$ is liquid viscosity and density, respectively. In the inertia dominated flow regime $(E O>40)$, the terminal rise velocity of large bubbles $U_{B}$ depends on the square root of the product of tube diameter $D$ and gravitational acceleration $g$.

$$
U_{B} \cong 0.351 \sqrt{g D}
$$

A significant increase in this terminal velocity is observed for the bubble rising in inclined tubes of medium sizes (see e.g. White and Beardmore [2], Zukolski [4], or Bendiksen [5]). However, the effect of tube inclination on the large bubble velocity is not completely understood, especially the role of surface and viscous forces taking place for small inclinations.

The recent development on microfluidic devices brings about the interest in two-phase flows in channels

a tihon@icpf.cas.cz with noncircular (usually rectangular) cross-sections. The first experiments on large flat bubbles were done by Griffith [6] and Collins [7]. They found the relationship qualitatively similar to Eq.1, just with the constant depending on the particular channel geometry. Sadatomi and Sato [8] studied experimentally the two-phase flow in vertical channels of various cross-sections and suggested the equi-perimeter radius $\left(R_{e}=P / 2 \pi\right)$ as a suitable scaling parameter for the terminal bubble velocity. This scaling has been recently supported by the analysis by Clanet et al. [9].

The experiments on flat bubbles rising at inclinations are very scarce. Maneri and Zuber [10] described two competing effects acting on the large bubbles at inclinations: streamlining the bubble shape increases the terminal velocity, whereas decreasing the axial component of buoyant force acts on the contrary. The two-phase flow patterns observed for the surface tension dominated flow of elongated bubbles in microchannels are discussed in the review by Kreutzer et al. [11]).

The objective of the present work is to study the motion of isolated large air bubbles in inclined flat channels (rectangular cross sections with high aspect ratios). Visual observations of the bubble motions done by a high-speed camera provide characteristic shapes and velocities of rising bubbles. The corresponding wall shear stress traces recorded by electrodiffusion sensors are then analyze to obtain detail information on the near-wall flow region. 


\section{Laboratory experiments}

\subsection{Experimental set-up}

The experimental flow channel made of Plexiglass is mounted on a positioning plate, whose inclination can be adjusted from the horizontal to vertical orientation. Its length is kept constant $(L=800 \mathrm{~mm})$ and the rectangular cross-section modified to obtain three configurations: with channel heights of $H=8,4$, and $1.5 \mathrm{~mm}$ and widths of $W=100,50$, and $50 \mathrm{~mm}$, respectively. The channel perimeters $(P=0.216,0.108$, and $0.103 \mathrm{~m}$, respectively) make possible to investigate the motion of large flat bubbles with different face profiles. Water with a small addition of electrochemical agents, used in our experiments as a model liquid, is supplied by a gear pump Heidolph 5230. The liquid flow rate (in upward direction) is ranging from 0.2 to $1.2 \mathrm{l} / \mathrm{min}$, corresponding to the liquid Reynolds number range from 30 to 400 (laminar flow regime). Air supplied from the institute's distribution system is filtrated and then fed into the channel through a needle located at a distance of $35 \mathrm{~mm}$ from the liquid inlet distributor. The needle $(0.65 \mathrm{~mm}$ i.d.) is glued into a mounting tube, which is passing through the roof wall of experimental channel. The bubbles growth is controlled by two electromagnetic valves (Burkert 6011). The pressure, opening time, and possible venting into the atmosphere are the parameters, which determine the size of air bubbles. The valves are controlled by digital outputs of a data acquisition board (National Instruments PCI-6221). The volume of bubbles rising in stagnant liquid is determined from the change of liquid level in a burette tube mounted at the channel output.

Two directionally sensitive, double electrodiffusion probes are applied to measure wall shear stress along rising bubbles and to detect the eventual reverse flow in a thin liquid film between the bubble and the channel wall. They are mounted into the roof wall of the channel at the distances of 460 and $660 \mathrm{~mm}$ from the needle, thus at the locations where steady bubble movement is assumed. The terminal velocity of bubbles can be then determined from the cross-correlation analysis of measured probe signals. This analysis provides the time necessary for a bubble to pass between two wall-mounted probes.

The movement of bubbles in the flow cell is observed by a high-speed camera (Redlake Motion Pro X3), typically at a rate of 1000 fps. The bubbles in their bottom view (field of view $10 \times 12 \mathrm{~cm}$ ) are observed in a mirror, which is located under the first probe and inclined $45^{\circ}$ in respect to the bottom channel wall. Our own software written in LabView software ensures the synchronization between electrodiffusion measurements and high-speed camera recordings.

\subsection{Electrodiffusion technique}

The electrodiffusion method (see Hanratty and Campbell [12] for a review), based on the measurement of the limiting diffusion current of the ferricyanide ions reduction at a small working electrode, is used to provide information on the wall shear rate under the moving bubble. The current signal $I$ provided by a simple strip probe is controlled by convective diffusion and depending on the wall shear rate $\gamma$ according to the following formula

$$
I=0.807 n F c_{0} w l^{2 / 3} D^{2 / 3} \gamma^{1 / 3}
$$

where $n$ is the number of electrons involved in the electrochemical reaction, $F$ is the Faraday constant, $l$ is the length of the strip in the mean flow direction, $w$ is its width, $c_{0}$ is the bulk concentration of the active ions, and $D$ is their diffusivity in the solution.

The directionally sensitive, double probe are fabricated from platinum foils. Their identical sensor segments (strips with sides of $l=0.1 \mathrm{~mm}$ and $w=1 \mathrm{~mm}$ ) are oriented with the longer side $\mathrm{w}$ perpendicular to the mean flow direction. An insulating gap between these segments has a thickness of $10 \mu \mathrm{m}$. The active segments are glued into a stainless steel tube (outside diameter of $5 \mathrm{~mm}$ ) serving as an anode. The near-wall flow reversal can be detected simply by comparing the magnitudes of current signals obtained from the upstream $\left(I_{l}\right)$ and downstream $\left(I_{2}\right)$ probe segment. The rear segment is in a concentration shade of the front one and thus it gives the lower signal. Thorough discussion on the application of double electrodiffusion probes (especially analysis of their dynamic behavior) can be found in Tihon et al. [13].

The fluid used for the experiments is water containing equimolar $0.025 \mathrm{M}$ potassium ferro/ferricyanide (as a suitable electrochemical system) and 1 wt.\% potassium sulfate (as a supporting electrolyte). The solution has a density of $\rho=1019 \mathrm{~kg} / \mathrm{m}^{3}$ and a kinematic viscosity of $v=1.064 \mathrm{~mm}^{2} / \mathrm{s}$ at a reference temperature of $21^{\circ} \mathrm{C}$. The polarization voltage of $-0.8 \mathrm{~V}$ is applied to assure that the probes are working under cathodic limiting diffusion current conditions. A homebuilt electrodiffusion device is used to apply the polarization voltage, to convert currents through the individual probe segments into voltages, and to amplify the resulting signals. A computer is used to control the electrodiffusion interface and data acquisition.

\section{Results and discussion}

For each configuration of the experimental channel $(H=8,4$, and $1.5 \mathrm{~mm})$, three operation parameters were adjusted in the following ranges: the volume of supplied air $V_{B}$ (from 1 to $80 \mathrm{ml}$ ), the liquid flow rate $Q_{L}$ (from 0 to $1.2 \mathrm{l} / \mathrm{min}$ ), and the slope of the flow cell $\alpha$ (from horizontal $\alpha=0^{\circ}$ to vertical $\alpha=90^{\circ}$ orientation).

\subsection{Bubble shape}

As the volume of air supplied into the experimental channel is increased, the shape of created bubbles significantly changes. Only very small bubbles preserve the spherical shape and do not feel the presence of limiting walls. A series of bottom-view photographs in Figure 1 shows the shapes of moderate size bubbles rising in stagnant liquid inside the inclined channel 
$\left(H=8 \mathrm{~mm}, \alpha=45^{\circ}\right)$. A gradual transition from a "spherical cap-like" shape at moderate bubble volumes to a "bullet-like" (so-called Taylor) shape at large bubble volumes is clearly visible. At the same time the bubble aspect ratio $\left(L_{B} / W_{B}\right.$, defined as a proportion between the bubble length $L_{B}$ and width $W_{B}$ ) is increasing (from 0.33 for Figure la to 1.14 for Figure 1d) and the bubble terminal velocity $U_{B}$ is quickly approaching a constant value. The bubble velocity $U_{B}=0.224 \mathrm{~m} / \mathrm{s}$, determined from high-speed video records for Figure 1c-d, is further independent of the bubble length. The ED probe flushmounted in the middle of the top wall is also seen in this figure.
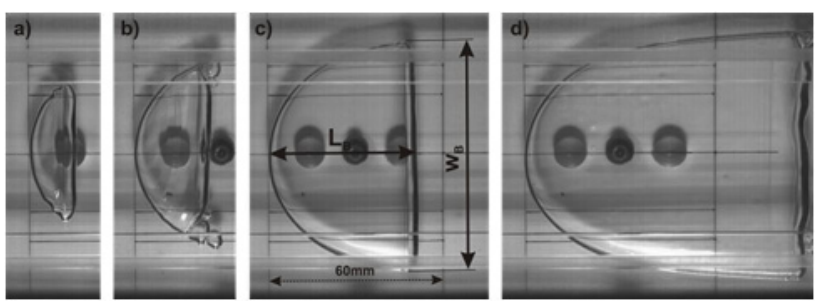

Fig. 1. The shapes of large bubbles rising in stagnant liquid. $\left(H=8 \mathrm{~mm}, V_{B}=4(\mathrm{a}), 8.5(\mathrm{~b}), 22(\mathrm{c})\right.$, and $\left.52 \mathrm{ml}(\mathrm{d})\right)$

As the bubble is enlarged its width grows and quickly reaches a level of $W_{B} / W \sim 0.7$, after that the bubble starts to "feel" the side walls and approaches the limit value ( $W_{B} / W \sim 1$, i.e. a possible contact with side walls) more slowly. The bubble elongation (increase in the axial size $L_{B}$ ) goes together with the bubble enlargement. The bubble aspect ratio $L_{B} / W_{B}$ first decreases (widening of small ellipsoidal bubbles), reaches the minimum value ( $L_{B} / W_{B} \sim 1 / 3$ corresponds to the shape from Figure 1a), then its value increases almost linearly (in the regime of "spherical cap-like" bubbles). At the same time, the rapid achievement of a constant terminal velocity is observed. In this case, the terminal velocity is measured already for bubbles with $V_{B}>10 \mathrm{ml}$ (i.e. for $W_{B} / W \sim 2 / 3$ or $\left.L_{B} / W_{B} \sim 1 / 2\right)$. The movement of small bubbles is quite unstable and exhibits strong oscillations, which impose a large scatter on velocity values measured for $V_{B}<5 \mathrm{ml}$.

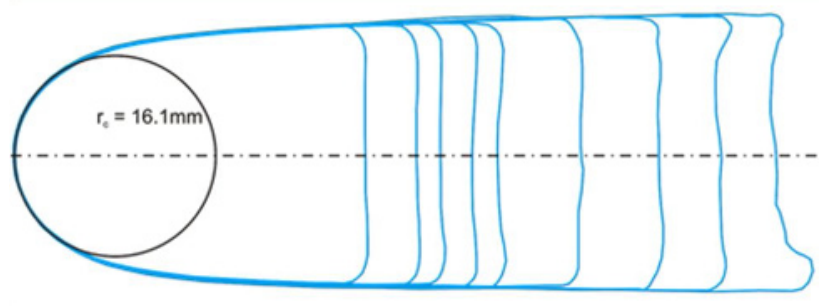

Fig. 2. The contour view of bubbles with the different lengths.

Increasing the volume of supplied air $V_{B}$ brings about the proportional elongation of large bubbles, but the face profile of these bubbles remains unchanged. This feature of general bubble outline is documented in Figure 2, where the contours of bubbles of different sizes are presented. These contours represent bubbles rising in the smallest channel $(H=1.5 \mathrm{~mm})$ inclined at the angle of $\alpha=45^{\circ}$. The frontal radius $r_{c}$ of all large bubbles depicted in Figure 2 is fixed at a value of $16.1 \mathrm{~mm}$. The same value is observed also if the liquid is not stagnant, but co-flowing with rising bubbles. The frontal radius of large bubble is thus control mainly by the channel size and can be quite well estimated from the value of channel equi-perimeter radius $\left(R_{e}=P / 2 \pi=(H+W) / \pi=16.4 \mathrm{~mm}\right)$.

Gravitation flattening of large bubbles is apparent especially at small inclination angles. As the channel slope is declined towards the horizontal position, the bubble frontal radius is slightly increasing and the bubble tail is approaching the channel side walls. However, as the experimental channel becomes thinner this flattening effect weakens (the viscous effects dominate the gravitation ones). The enlargement of bubble outline with the reducing of channel inclination is documented in Figure 3, where the results obtained in our largest channel $(H=8 \mathrm{~mm})$ are presented. As the volume of the compared bubbles is constant $\left(V_{B} \sim 50 \mathrm{ml}\right)$, it is evident that the bubble side-view profile (not visualized in this work) has to be also strongly modified with changing the channel slope.

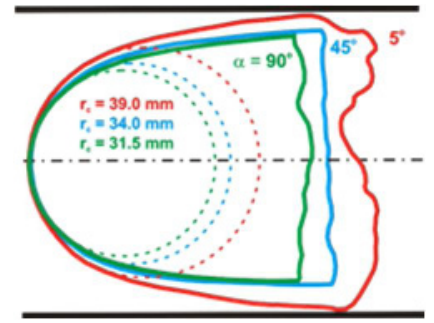

Fig. 3. The change of bubble frontal radius with the channel inclination angle.

\subsection{Bubble velocity}

The dependence of terminal bubble velocity on the channel inclination is shown in Figure 4. As the axial

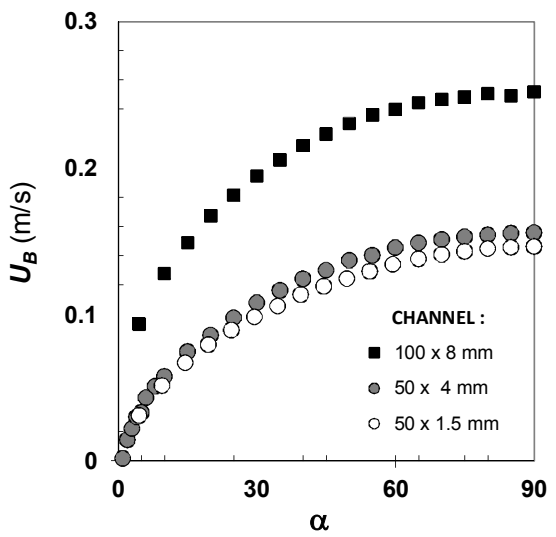

Fig. 4. The dependence of the velocity of Taylor bubbles on the channel inclination angle.

component of gravitation $(g \cdot \sin \alpha)$ is decreased along with the inclination angle, the bubble velocity is diminished. This trend is qualitatively different from those previously observed for the motion of long bubbles in inclined tubes (see Zukolski [4] or Bendiksen [5]), where the maximum bubble velocity was measured at inclinations about $\alpha \sim 45^{\circ}$. The comparison of velocity data for three channel 
configurations demonstrates that the velocity of large bubbles in flat channels is control mainly by the dimension of larger channel size (i.e. by the channel width).

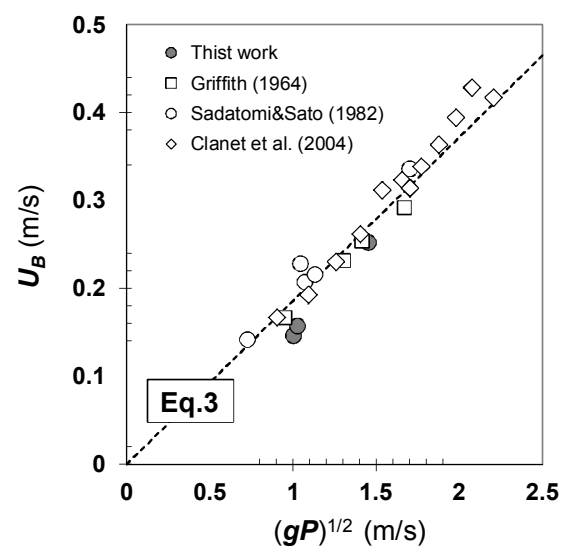

Fig. 5. The velocity scaling for Taylor bubbles in vertical rectangular channels.

In Figure 5 velocity data measured by the different authors in vertical rectangular channels are compared with the aim to confirm the velocity scaling based on the channel perimeter $P$

$$
F r=U_{B} / \sqrt{g P} \cong 0.2,
$$

which has been suggested by Clanet et al. [9]. Eq.3 follows the velocity data quite satisfactory; although a constant of 0.19 fits better all experimental values.

The velocity of bubble translation in co-flowing liquid $U_{B}$ can be expressed as a sum of two components; the bubble velocity in stagnant liquid $U_{B o}$ plus a component due to the liquid motion:

$$
U_{B}=U_{B o}+C_{0} U_{L}
$$

This linear relationship was introduced by Nicklin et al. [3] with a constant $C_{0} \sim 1.2$ obtained experimentally for the turbulent flow regime. Figure 6 (data for the channel with $H=4 \mathrm{~mm}$ ) demonstrates that a linear dependence between the rising velocity of bubbles $U_{B}$ and the mean liquid velocity $U_{L}$ holds also for the laminar flow regime in inclined rectangular flow channels.

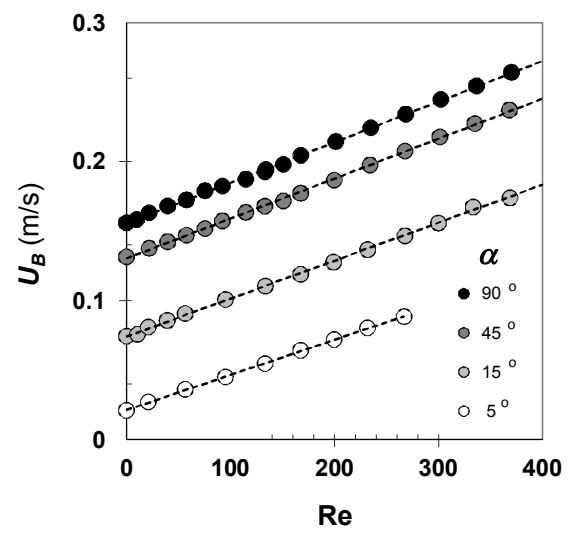

Fig. 6. The velocity of large bubbles rising in co-flowing liquid.
The coefficient $C_{0}$ is found to be sensitive to the channel size and inclination (slightly increasing with both these parameters). For our experimental data, $C_{0}$ is then ranging from 1.0 to 1.4 .

\subsection{Wall shear rate}

Typical electrodiffusion signals measured simultaneously with high-speed recording are shown in Figure 7. The primary current signals measured during the pass of single bubble between two electrodiffusion probes (black lines for $x=440 \mathrm{~mm}$ and grey lines $x=640 \mathrm{~mm}$; thick lines for front segments $I_{1}$, thin lines for rear segments $I_{2}$ of probes) are presented in Figure 7a. The calculated time series of wall shear rate corresponding to these primary data are seen in Figure $7 b$.
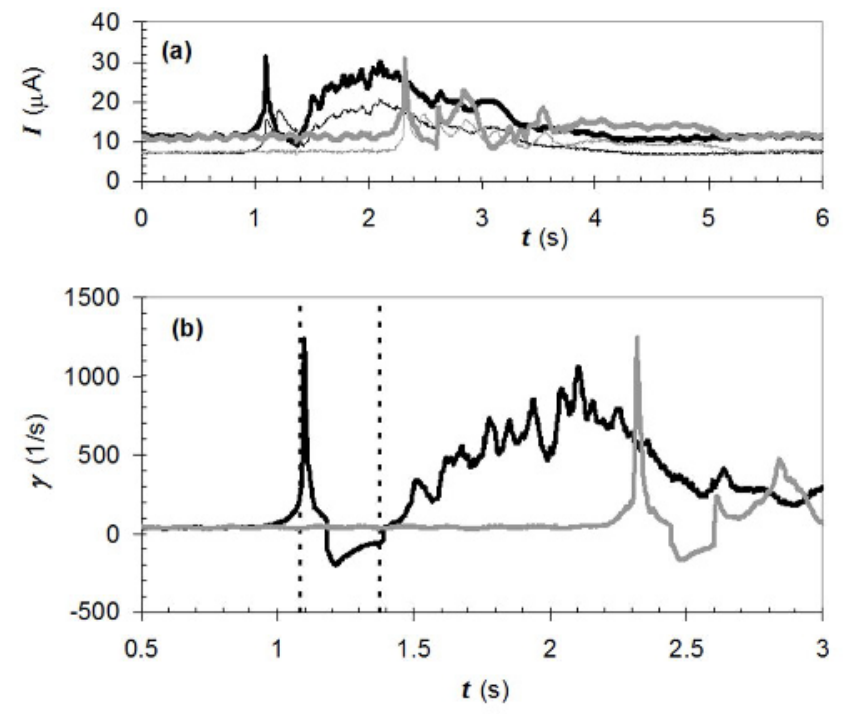

Fig. 7. The probe response to a passing bubble: (a) measured current signals, (b) wall shear rate.

The regions of steady single phase flow $(t=0 \div 1$ and $5 \div 6 \mathrm{~s})$, flow enhancement in front of the bubble $(t \sim 1.1$ and $2.3 \mathrm{~s}$ ), reverse flow inside the liquid film under the bubble $(t=1.2 \div 1.4$ and $2.4 \div 2.6 \mathrm{~s})$, and also unsteady flow in the bubble wake $(t=1.4 \div 4$ and $2.6 \div 5.2 \mathrm{~s})$ can be recognized in the current or wall shear rate signals. The dashed lines in Figure $7 \mathrm{~b}$ designate the time instants of the arrival of bubble nose at the first probe $(t \sim 1.08 \mathrm{~s})$ and the departure of bubble tail from it $(t \sim 1.38 \mathrm{~s})$. Consequently, the time of bubble pass along the probe is $\Delta t \sim 0.3 \mathrm{~s}$. The time necessary for this bubble to pass the distance between two wall probes $\left(x_{12}=200 \mathrm{~mm}\right)$ corresponds to the time shift observed between the peaks on two wall shear rate signals $\left(t_{12}=1.22 \mathrm{~s}\right)$. The averaged bubble velocity $\left(U_{B}=x_{12} / t_{12}=0.164 \mathrm{~m} / \mathrm{s}\right)$ calculated from these signals is in a good agreement with that obtained from the video movie recording the bubble moving around the first probe. Consequently the bubble motion between two probes is steady, with a terminal rising velocity.

The analysis of wall shear rate data for extremely long bubbles provides also valuable information on the liquid film flow, which is formed between the bubble and the 
channel wall. The steady negative values of wall shear rate are recorded under the rear part of long bubbles. For the vertical channel configuration, these values are controlled by the liquid film flowing freely (under the effect of gravitation) along the wall. The proportional relationship between the wall shear rate $\gamma$ and the film thickness $h$ gives the formula for film thickness estimation

$$
h=\frac{\mu}{g \rho} \gamma
$$

The values of $h$ ranging from 5 to $400 \mu \mathrm{m}$ are thus provided for long bubbles rising in vertical rectangular channels with different flow configurations.

Another interesting feature observed on the liquid film separating the large bubble from the wall is the existence of capillary waves. The picture of this phenomenon is shown in Figure 8, where the capillary waves with a length of $\lambda_{w}=1.5 \mathrm{~mm}$ are easily distinguished. As the measured bubble terminal velocity in this flow case is $U_{B}=0.252 \mathrm{~m} / \mathrm{s}$, the frequency of observed capillary waves is $f_{w}=168 \mathrm{~Hz}$.

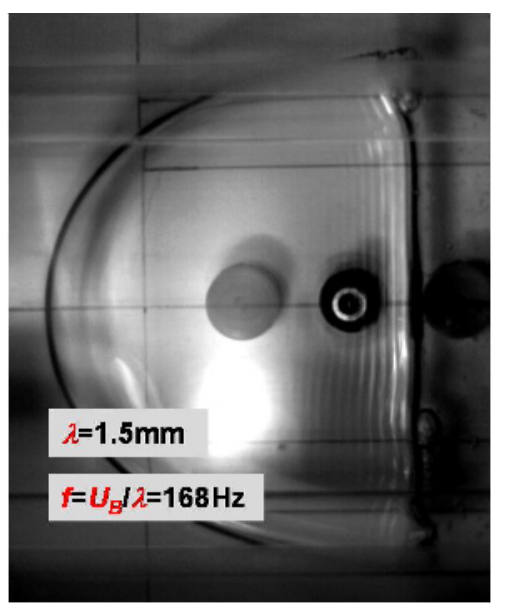

Fig. 8. Capillary waves appearing in the bubble tail region.

The same frequency can be also extracted from wall shear rate oscillations measured by the electrodiffusion probe in the capillary wave region. For vertical falling liquid film flow achieved at the bubble tail position, the wall shear rate measurement provides also information on the mean liquid film velocity

$$
U_{F}=\frac{\mu \gamma^{2}}{3 g \rho},
$$

which reaches in the case studied in Figure 8 a value of $U_{F}=-0.294 \mathrm{~m} / \mathrm{s}$. As the bubble is rising up and the liquid film is falling down, the relative velocity at the gas/liquid interface is quite high $\left(U_{R}=U_{B}-U_{F}=0.546 \mathrm{~m} / \mathrm{s}\right)$. If we insert this relative velocity into the Lighthill's formula for surface tension controlled ripples

$$
\lambda_{w}=\frac{2 \pi \sigma}{\rho U_{R}^{2}},
$$

we calculate the wave length $\lambda_{w}=1.48 \mathrm{~mm}$ that agrees very well with the experimental one. Therefore the electrodiffusion technique is able to detect capillary waves and determine correctly their characteristics.

Similarly as in the case of bubble shapes, also the wall shear rate traces corresponding to passing of large bubbles are self-similar. The wall shear rate profiles measured for the several bubble volumes ( $V_{B}$ from 3 to $28 \mathrm{ml}$ ) at the channel bottom wall (for $H=4 \mathrm{~mm}$, $\alpha=45^{\circ}$; in stagnant liquid) are shown in Figure 9. Here, the development of a falling liquid film under the bubble (reaching a constant negative value of $\gamma$ ) and a rim at the bubble back region (enlargement of a peak at $\gamma \sim-150 \mathrm{~s}^{-1}$ ) are clearly seen. Symmetrical bottom/top profiles are observed only in vertical channel configurations. A liquid film separating in inclined channels the bubble from the top wall is thinner than that at the bottom wall, therefore the positive peak of wall shear rate observed at a bubble front is higher and the negative level due to reverse film flow is lower there.

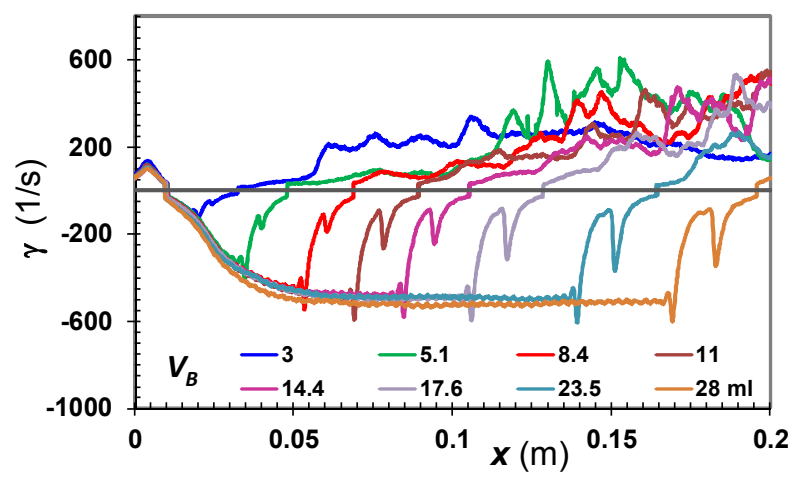

Fig. 9. Wall shear rate profiles: effect of the bubble size ( $H=4 \mathrm{~mm}, \alpha=45^{\circ}, U_{L}=0 \mathrm{~m} / \mathrm{s}$, bottom wall).

The effect of channel inclination on the wall shear rate profiles measured at the top wall is evident (see Figure 10). As the channel is raised up towards the vertical orientation, the negative value of wall shear rate in the liquid film region (i.e. the level of reverse flow) is gradually augmented. On the other hand, at the bottom wall, where the reverse flow in liquid film is stronger (especially at small channel inclinations), the situation is different: the maximum value is usually observed at moderate inclinations $\left(\alpha \sim 50^{\circ}\right)$.

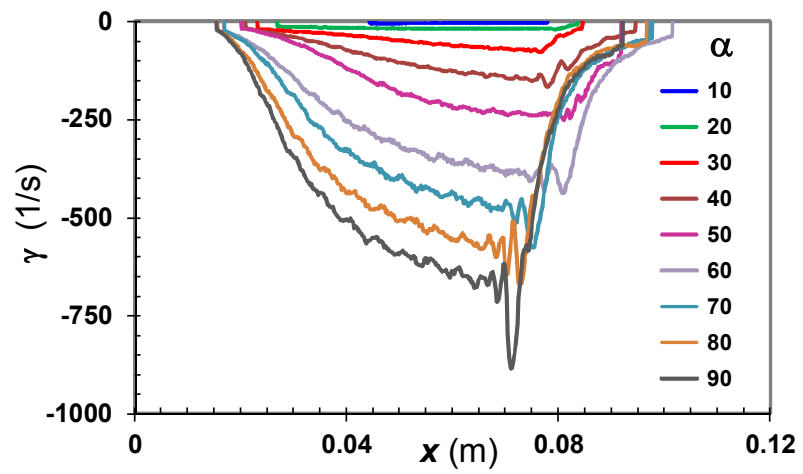

Fig. 10. Wall shear rate profiles: effect of the inclination angle ( $H=8 \mathrm{~mm}, U_{L}=0 \mathrm{~m} / \mathrm{s}, V_{B} \cong 25 \mathrm{ml}$, top wall). 
As seen in Figure 11, the intensification of reverse flow in the liquid film above the bubble can be achieved also by the increasing flow rate of co-flowing liquid. At the same time, the thickening liquid film is observed. As the effect of co-flowing liquid is stronger at the top wall, the bubbles rising in inclined channels are consequently pushed towards the centerline position.

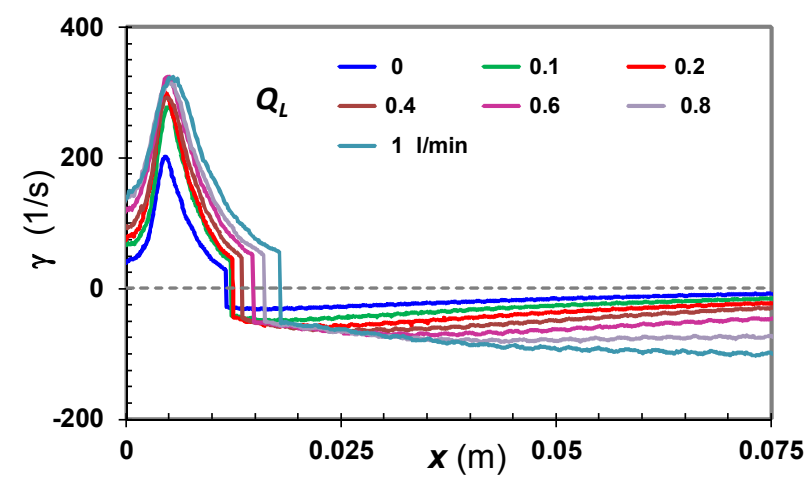

Fig. 11. Wall shear rate profiles: effect of the liquid flow rate ( $H=4 \mathrm{~mm}, \alpha=15^{\circ}, V_{B} \cong 25 \mathrm{ml}$, top wall).

\section{Conclusions}

The experiments on Taylor bubbles rising in stagnant or co-flowing liquid provided some new results on the bubble shape, the bubble velocity and the wall shear rate brings along by bubbles in inclined rectangular channels.

The frontal radius of large bubbles is found to be independent of the bubble size and it is not also affected by the upward liquid flow in the channel. The gravitation flattening of large bubbles observed at small inclination angles leads to significant bubble shape and velocity changes.

The velocity scaling suggested by Clanet et al. [9] for the rise of large bubbles in non-circular channels (Eq.3) is confirmed. The linear relationship between the bubble velocity and the mean velocity of co-flowing liquid (Eq.4) is find to hold also for the bubble rise in inclined rectangular channels.

The electrodiffusion technique proved to be an effective tool to investigate the near-wall flow response to moving bubbles. The two-strip probe flush-mounted into the channel wall provides information not only on the wall shear rate profile along the passing bubble, but also gives an estimate of the thickness of liquid film and the frequency of possible capillary waves on this film.

\section{Acknowledgement}

The support by Czech Science Foundation GACR through the contract $\mathrm{P} 101 / 12 / 0585$ is gratefully acknowledged.

\section{References}

1. D.T Dumitrescu, ZAMM 23, 139(1943) 139-149.

2. E.T. White, R.H. Beardmore, Chem. Eng. Sci. 17, 351 (1962)
3. D.J. Nicklin, J.O. Wilkes, J.F. Davidson, Trans. Instn. Chem. Engrs. 40, 61 (1962)

4. E.E. Zukoski, J. Fluid Mech. 25, 821 (1966)

5. K.H. Bendiksen, Int. J. Multiphase Flow 10, 467 (1984)

6. P. Griffith, J. Heat Transfer 86, 327 (1964)

7. R. Collins, J. Fluid Mech. 22, 763 (1965)

8. M. Sadatomi, Y. Sato, J. Multiphase Flow 8, 641 (1982)

9. C. Clanet, P. Heraud, G. Searby, . J. Fluid Mech. 19, 359 (2004)

10. C.C. Maneri, N. Zuber, Int. J. Multiphase Flow 1, 623 (1974)

11. M.T. Kreutzer, F. Kapteijn, J.A. Moulijn, J.J. Heiszwolf, Chem. Eng. Sci. 60, 5895 (2005)

12. T.J. Hanratty, J.A. Campbell, Fluid mechanics measurements (Washington, Hemisphere, 1983)

13. J. Tihon, V. Tovchigrechko, V. Sobolik, O. Wein, J.Appl.Electrochem. 33, 577 (2003) 